\title{
SPHERICAL IMAGES OF CONVEX SURFACES
}

\author{
M. DO CARMO AND B. LAWSON
}

Let $x: M^{n} \rightarrow R^{n+1}$ be an isometric immersion of an orientable, complete riemannian manifold $M^{n}$ (superscripts denote dimension) into the euclidean space $R^{n+1}$ and let $\nu: M^{n} \rightarrow S^{n} \subset R^{n+1}$ be the normal (Gauss) map of $x$. Assume that the surface $x(M)$ is convex in $R^{n+1}$, i.e., $x(M)=\partial K$, where $K \subset R^{n+1}$ is a convex set with interior points. It has-been long believed that the spherical image $v(M)$ is then convex in $S^{n}$ (cf. [1, p. 25]). Recently, $\mathrm{H}$. Wu [2] provided a counterexample to this assertion and proved the weaker statement:

THEOREM. The closure of $v(M)$ is convex in $S^{n}$.

Wu's proof is rather long and is designed to work in the more general context of continuous convex surfaces. We present here a simple proof for the differentiable case. We remark that the proof works for the infinite dimensional case as well.

Proof of The Theorem. Consider first the case where $v_{0}=v\left(p_{0}\right)$ and $v_{1}=v\left(p_{1}\right)$ are two nonantipodal points of $S^{n}$. Let $v$ be a point in the smallest arc of $S^{n}$ joining $v_{0}$ to $v_{1}$. Fix an orientation on $M$ in such a way that the normals to $x(M)$ point towards $K$, and consider the height function $h: M \rightarrow R$ defined by $h(p)=\langle x(p), v\rangle, p \in M$. By convexity, $h$ is bounded below. Let $p \in M$ and $\varphi(t)$ be the trajectory of $-\operatorname{grad} h$ with $\varphi(0)=p$. Since $\|\operatorname{grad} h\| \leqq 1$ and $M$ is complete, $\varphi(t)$ is defined for all $t \in(-\infty, \infty)$. We claim that $\|\operatorname{grad} h(\varphi(t))\|$ is not bounded away from zero in $[0, \infty)$. Otherwise

$$
\lim _{t \rightarrow \infty} h(\varphi(t))-h(\varphi(0))=-\int_{0}^{\infty}\|\operatorname{grad} h(\varphi(t))\|^{2} d t=-\infty,
$$

which contradicts the fact that $h(\varphi(t))$ is bounded below.

It follows that either there is a critical point $\varphi\left(t_{0}\right)$ of $h, t_{0} \in[0, \infty)$, and then the normal vector at $\varphi\left(t_{0}\right)$ is $v$, or there exists a sequence of points in $M$ whose normals converge to $\nu$. In any case, $\nu$ belongs to the closure of $v(M)$.

Received by the editors July 21, 1971 .

AMS 1970 subject classifications. Primary 53C40, 52A20.

Key words and phrases. Convex surface, Gauss map, spherical image.

(c) American Mathematical Society 1972 
The remaining cases are simpler. If some or both $v_{0}$ and $v_{1}$ belong to the boundary of $v(M)$, and are not antipodal, we take the limit of minimal geodesics joining $\boldsymbol{v}_{n}^{\mathbf{0}}$ to $\boldsymbol{v}_{n}^{\mathbf{1}}, n=1, \cdots$, where $\left\{\boldsymbol{v}_{n}^{\mathbf{0}}\right\} \rightarrow v_{0},\left\{\boldsymbol{v}_{n}^{\mathbf{1}}\right\} \rightarrow \boldsymbol{v}_{1}$, and no pair $v_{n}^{0}, v_{n}^{1}$ is antipodal. If $v_{0}$ and $v_{1}$ belong to the closure of $v(M)$ and are antipodal, we consider a third point $v_{2}=v\left(p_{2}\right)$, distinct from $v_{0}$ and $v_{1}$ (which exists because $M$ is connected), and prove easily that the smallest triangle $v_{0} v_{1} v_{2}$ belongs to the closure of $v(M)$.

\section{REFERENCES}

1. H. Busemann, Convex surfaces, Interscience Tracts in Pure and Appl. Math., no. 6, Interscience, New York, 1958. MR 21 \#3900.

2. $\mathrm{H}$. Wu, A structure theorem for complete noncompact hypersurfaces of nonnegative curvature, Berkeley preprint.

Instituto de Matemática Pura e Aplicada (IMPA), Rio de Janeiro, Brasil 